\title{
METÁSTASIS CEREBRALES EN CÁNCER DE PULMÓN DE CÉLULAS NO PEQUEÑAS CON MUTACIÓN EGFR
}

\author{
CEREBRAL METASTASES IN LUNG CANCER OF NON-SMALL CELLS WITH EGFR MUTATION \\ Robert Malpartida-Palomino', Rómulo Cárdenas-Agramonte1,2,a
}

\begin{abstract}
RESUMEN
El cáncer de pulmón es uno de los tumores que tienen la capacidad de metastatizarse en el cerebro y tienen la capacidad de penetrar la barrera hematoencefálica, la clínica es variada dependiendo del sitio afectado, así como los riesgos asociados de convulsión. Por lo tanto, la elección del tratamiento es compleja, considerando el primario, el número de metástasis y los sitios afectados. La radioterapia ha sido durante mucho tiempo la elección de los pacientes que no son candidatos a la cirugía, La presencia de mutaciones han permitido brindar terapias dirigidas como los inhibidores tirosin kinasas que penetran la barrera hematoencefálica y han sido clave para el manejo terapéutico.
\end{abstract}

Palabras clave: Cáncer de pulmón; Metastasis cerebral; EGFR; Mutación EGFR. (fuente: DeCS BIREME)

\begin{abstract}
Lung cancer is one of the tumors that have the ability to metastasize in the brain and have the ability to penetrate the blood-brain barrier, the clinic is varied depending on the affected site, as well as the associated risks of seizure. Therefore, the choice of treatment is complex, considering the primary, the number of metastases and the affected sites. Radiotherapy has long been the choice of patients who are not candidates for surgery. The presence of mutations have allowed targeted therapies such as tyrosine kinase inhibitors that penetrate the blood-brain barrier and have been key to therapeutic management.
\end{abstract}

Key words: Lung cancer; Brain metastases; EGFR; Mutation EGFR. (source: MeSH NLM)

\section{INTRODUCCIÓN}

El cáncer de pulmón es la principal causa de muerte en todo el mundo, tanto en hombres como en mujeres. En el Perú es la quinta neoplasia detectada con prevalencia $6 \times 100,000$ habitantes con edad promedio 60 años y la prevalencia del sexo masculino 2,074 (67.1\%) femenino 1,015 (32.9\%) y su relación (M/F) 2.5/1 donde el principal factor de riesgo que se encontró es el tabaquismo en $75 \%$ de los casos. De los cuales los estadios clínicos estadío I 3.5\%, estadío II 4.4\%, estadío III 32.4\% y estadío IV 59.7\%. (Fuente: Registro de cáncer de Lima Metropolitana Inst. Investigación Maes Heller. INEN-2013). El cáncer pulmón se puede clasificar en el de células no pequeñas $C P C N P$ y células pequeñas (CPCP). Desde el punto de vista histológico encontramos una variedad entre la que predomina el adenocarcinoma. El CPCNP representa aproximadamente el 85 por ciento de los cánceres de pulmón, y el resto como cáncer de pulmón de células pequeñas CPCP ${ }^{1}$. Los pacientes portadores de CPCNP con histología adenocarcinoma presentan mutaciones moleculares: La activación de la mutación en el receptor del factor de crecimiento epidérmico (EGFR) que se presenta en pacientes no fumadores, sexo femenino y de etnia asiática. La presencia del oncogén de fusión del linfoma quinasa anaplásico (ALK) que son más frecuentes en no fumadores o exfumadores y se produce a una edad más temprana. Existen otras mutaciones menos frecuentes como BRAF, HER2, $\beta$-catenina, DDR2 y MEK. La expresión del tumor PD-L1 predice la respuesta a ciertas inmunoterapias

\footnotetext{
' Servicio de Oncología Médica, Hospital Militar Central, Lima-Perú.

${ }^{2}$ Consultor Asesor Docente del Hospital Militar Central, Lima-Perú.

a Especialista en Oncología Médica.
}

Citar como: Robert Malpartida-Palomino, Rómulo Cárdenas-Agramonte. Metástasis cerebrales en cáncer de pulmón de células no pequeñas con mutación EGFR. [Caso Clínico].2019;19(1):101-104. (Enero 2019). DOI 10.25176/RFMH.v19.n1.1798 
y puede guiar la elección del tratamiento tanto en el tratamiento de primera línea como en el de la línea subsiguiente ${ }^{2,3}$. Las metástasis cerebrales son una complicación común en una amplia gama de cánceres, pero son particularmente comunes entre los pacientes con cáncer de pulmón. Los pacientes con metástasis cerebrales, el cáncer de pulmón es el tumor primario en 40 a 50 por ciento de los casos y aproximadamente el 10 por ciento de los pacientes recién diagnosticados con cáncer avanzado de CPCNP tienen metástasis cerebrales $^{3,4}$ Las metástasis cerebrales son una causa común de morbilidad y mortalidad en pacientes con CPCNP. Los pacientes que presentan mutación EGFR tienen mejor pronóstico debido a la supervivencia prolongada de los agentes sistémicos dirigidos 5 . En el 2016 un estudio revelo que la mediana de supervivencia global (OS) de una población portadora de CPCNP con metástasis cerebral que se dividió en 2 grupos: EGFR- no mutado y un EGFR mutado, varió de 3 a 15 meses $^{6}$. La sobrevida global (SG) en pacientes CPCNP con metástasis cerebral y EGFR mutado se observó que va de 19-58 meses ${ }^{7,8}$. Sin embargo, los estudios previamente publicados que describen el uso terapia combinada de cisplatino y de pemetrexed presentan buena tolerabilidad ${ }^{9,10}$. Durante la última década, los inhibidores de EGFR-tirosina quinasa (TKI) se han empleado con éxito en pacientes con CPCNP basándose en la identificación de mutaciones del gen EGFR ${ }^{11,12}$. Otros estudios mostraron que la combinación de RT y EGFR-TKI produjo resultados superiores para pacientes con CPCNP con metástasis cerebral y mutaciones de EGFR ${ }^{13,14}$.

\section{REPORTE DE CASO}

Paciente de 54 años de sexo femenino, nacida en Huancayo, procedente de Lima ingresa por emergencia del Hospital Militar Central el día 12/09/15 con un tiempo de enfermedad de 1 mes caracterizado convulsiones tónico clónico. Antecedentes personales y familiares: Exposición a la leña por más de 20 años. Niega alergia a medicamentos. Antecedentes quirúrgicos: Colecistectomizada hace 14 años.

Examen Físico: ECOG 3-4. Piel y mucosas: palidez $+/+++$, ictericia (-), mucosas secas++/+++, no edemas. Fascies abotagada. Auscultación cardiopulmonar: Murmullo vesicular pasa bien por ambos hemitorax no ruidos agregados. Abdomen: ruidos hidroaereos (+), blando, depresible, no masas, no visceromegalia. Genitourinario: Puño percusión lumbar (-) no hematuria, no disuria, no globo vesical. Neurológico: desorientada con tendencia al sueño y marcha inestable.

\section{EXÁMENES AUXILIARES:}

- Marcadores Tumorales:

\begin{tabular}{cccccccc} 
MARCADORES & $\mathbf{1 4 / 0 9 / 1 5}$ & $\mathbf{1 5 / 0 3 / 1 6}$ & $\mathbf{2 1 / 0 6 / 2 1 6}$ & $\mathbf{2 5 / 0 8 / 1 6}$ & $\mathbf{2 2 / 1 1 / 1 6}$ & $\mathbf{2 3 / 2 / 1 7}$ & $\mathbf{2 3 / 0 5 / 1 7}$ \\
CEA & 81.6 & 4.9 & 1.9 & 1.9 & 2.9 & 7.9 & 38.2 \\
CA125 & 83.8 & - & - & - & - & - & - \\
\hline
\end{tabular}

\section{Imágenes:}

- TEM CEREBRO (12/09/15): formaciones de aspecto nodular intracerebrales que se asocian a edema vasogénico perilesional ubicadas en la región frontoparietal izquierda y occipital derecha la última de ellas mide $25 \mathrm{~mm}$.

- RMN CEREBRO (14/09/15): tres lesiones focales dos de ellas de localización córtico-subcortical que comprometen el lóbulo occipital derecho y el lóbulo parietal izquierdo y una tercera que compromete el núcleo lenticular izquierdo. La lesión de mayor tamaño localizado en el lóbulo occipital derecho muestra intenso realce y se asocia a edema vasogénico perilesional, las otras dos muestran área de aspecto necrótico.
- TEM TORAX (18/09/15): Masa sólida de aspecto proliferativo de bordes irregulares en segmento 10 derecho de $37 \mathrm{~mm} \times 29 \mathrm{~mm}$. Ganglios mediastinales y axilares de hasta $8 \mathrm{~mm}$.

\section{PROCEDIMIENTO:}

\section{- BIOPSIA NÓDULO PULMONAR (5/11/15):}

AP: parénquima pulmonar infiltrado por nidos de neoplasia maligna de aspecto epitelial glandular con áreas de necrosis. IHQ: TTF-1: (+) NAPSINA: (+) CYTOKERATINA 7: (+) ADENOCARCINOMA PRIMARIO PULMONAR

Se realiza mutación: EGFR EXON $19(+)$

DIAGNÓSTICO DX: CANCER DE PULMÓN ESTADÍO CLÍNICO IV POR METASTASIS CEREBRAL 


\section{TRATAMIENTO:}

RADIOTERAPIA HOLOCRANEAL por síndrome hipertensión endocraneana.

10 SESIONES 300cGY dosis total: 3000cGY desde $(2 / 12 / 15$ al $15 / 12 / 15)$

Inicio tratamiento con ITK diciembre 2015 a dosis de 150 mg. /día de ERLONITIB.

\section{INTERCURRENCIAS:}

15/03/16: suspende de forma temporal tratamiento de ITK por presentar herpes zoster. 22/03/16 reanudar ITK.

\section{PRIMERA REEVALUACIÓN DE ENFERMEDAD:}

\section{TEM TÓRAX/ABDOMEN/PELVIS (09/04/16)} NEOPLASIA.

RMN CEREBRO: Lesiones focales con edema periférico de pequeño tamaño en el núcleo lenticular del lado izquierdo y en corteza frontal del mismo lado, otras de mayor tamaño en el lóbulo occipital de lado derecho.

\section{DIAGNÓSTICO RESPUESTA PARCIAL.}

\section{TRATAMIENTO:}

Continúa tratamiento con ITK ERLOTINIB 150 mg/día. SEGUNDA REEVALUCIÓN DE ENFERMEDAD:

TEM TORAX (11/11/16): en la porción central entre el segmento 7 y 9 derecho, imagen nodular sólida de 13 y $16 \mathrm{~mm}$.

\section{TEM ABDOMEN/PELVIS: (-) NEOPLASIA}

RMN CEREBRO 14/11/16: En el lóbulo derecho área medial o para sagital dos imágenes que disminuyen el volumen cortical, dependiente de edema circundante a dos pequeñas lesiones que resaltan en la periferia de $8 \mathrm{~mm}$ la de mayor tamaño.

\section{DIAGNÓSTICO RESPUESTA PARCIAL}

\section{3.- TRATAMIENTO:}

Continua tratamiento con ITK ERLOTINIB 150 mg. /día.

\section{TERCERA REEVALUACION DE ENFERMEDAD:}

TEM TORAX 19/02/17: masa pulmonar en segmento apical de LID que mide $42 \mathrm{~mm} \times 37 \mathrm{~mm}$ x $36 \mathrm{~mm}$ de bordes definidos de localización sub pleural.

DIAGNÓSTICO RESPUESTA: PROGRESION DE ENFERMEDAD.

\section{4.- TRATAMIENTO:}

Radioterapia externa en pulmón derecho campo anterior y posterior.

16 SESIONES 250cGY dosis total: 4000cGY desde (28/02/17 al 27/03/17)

\section{INTERCURRENCIAS:}

Radiodermitis VS dermatitis de contacto controlado por dermatología.

Hospitalización (02/05/17): cefalea, hemiparesia de lado izquierdo, astenia.

\section{CUARTA REEVALUACION DE ENFERMEDAD:}

TEM TORAX (05/05/17): Segmento basal posterior del LID nodulación sólida irregular de 21x22x $20 \mathrm{~mm}$. Bordes irregulares resto sin alteraciones.

TEM ABDOMEN/PELVIS: (-) NEOPLASIA

RMN CEREBRO (6/05/17): lesión a nivel occipital derecho de 25x28x $24 \mathrm{~mm}$. Se asocia extensas áreas con alteración de señal perilesional de distribución subcortical con desplazamiento de la línea media de $15 \mathrm{~mm}$ y disminución del volumen de ventrículo derecho.

DIAGNÓSTICO RESPUESTA: PROGRESIÓN DE ENFERMEDAD A NIVEL CEREBRAL LESIÓN ÚNICA.

\section{5.- TRATAMIENTO:}

Continua tratamiento con ITK ERLOTINIB 150 mg. /día. Inicio de Radioterapia re-irradiación de lesión única.

Radioterapia 12 sesiones desde 16 junio 2017 - 30 junio 2017.

\section{CONCLUSIÓN}

Las metástasis cerebrales es una complicación común en cáncer de pulmón. Para pacientes portadores de CPCNP con metástasis cerebral sin efecto de masa o riesgo de hernia a nivel cerebral y presenta mutación EGFR positivo, se debe iniciar terapias dirigidas con los ITK. En el caso que se presentó se evidencia que existe resistencia al TKI (erlotinib), esta paciente desarrollo progresión tanto intracraneal como extracraneal donde se solicitó toma de biopsia del sitio más accesible (por razones extramédicas no se realizó). La recomendación terapéutica es iniciar osimertinib ya que ha logra mayores concentraciones intracraneales y ha demostrado que tiene una actividad intracraneal significativa contra metástasis cerebrales a la dosis estándar de $80 \mathrm{mg}$ diarios, incluso contra la carcinomatosis leptomeníngea y aquellos con una mutación de resistencia T790M.

Contribuciones de autoría: Los autores participaron en la generación, recolección de información, redacción y aprobación final del artículo original.

Financiamiento: Autofinanciado.

Conflicto de interés: Los autores declaran no tener conflicto de interés en la publicación de este artículo.

Recibido: 28 de Agosto del 2018

Aprobado: 17 de diciembre del 2018 
Correspondencia: Robert Malpartida Palomino

Dirección: Condominio Golf Los Andes EDF 06 Departamento 101, Lima-Perú

Celular: +51981236407 1era. Etapa Naña-Chosica

Correo: robertmp7@hotmail.com

\section{REFERENCIAS BIBLIOGRÁFICAS}

1. Torre LA, Bray F, Siegel RL, y col. Estadísticas globales de cáncer, 2012. CA Cancer J Clin 2015; 65:87.

2. Pikor LA, Ramnarine VR, Lam S, Lam WL. Genetic alterations defining NSCLC subtypes and their therapeutic implications. Lung Cancer 2013; 82:179.

Takahashi T, Sonobe M, Kobayashi M, et al. Clinicopathologic features of non-small-cell lung cancer with EML4-ALK fusion gene. Ann Surg Oncol 2010; 17:889.

3 Barnholtz-Sloan JS, Sloan AE, Davis FG, et al. Incidence proportions of brain metastases in patients diagnosed (1973 to 2001) in the Metropolitan Detroit Cancer Surveillance System. J Clin Oncol 2004; 22:2865.

4 Schouten LJ, Rutten J, Huveneers HA, Twijnstra A. Incidence of brain metastases in a cohort of patients with carcinoma of the breast, colon, kidney, and lung and melanoma. Cancer 2002; 94:2698.

5. Shin DY, Na II, Kim CH, Park S, Baek H, Yang SH. EGFR mutation and brain metastasis in pulmonary adenocarcinomas. J Thorac Oncol. 2014;9(2):195-199.

6. Sperduto PW, Kased N, Roberge D, et al. Summary report on the graded prognostic assessment: an accurate and facile diagnosisspecific tool to estimate survival for patients with brain metastases. Clin Oncol. 2012;30(4):419-425.

7. Gerber NK, Yamada Y, Rimner A, et al. Erlotinib versus radiation therapy for brain metastases in patients with EGFR-mutant lung adenocarcinoma. Int J Radiat Oncol Biol Phys. 2014;89(2):322-329.
8. Magnuson WJ, Yeung JT, Guillod PD, Gettinger SN, Yu JB, Chiang VL. Impact of deferring radiation therapy in patients with epidermal growth factor receptor-mutant non-small cell lung cancer who develop brain metastases. Int J Radiat Oncol Biol Phys. 2016;95(2):673-679.

9. Barlesi F, Gervais R, Lena $H$, et al. Pemetrexed and cisplatin as first-line chemotherapy for advanced non-small-cell lung cancer (NSCLC) with asymptomatic inoperable brain metastases: a multicenter phase II trial (GFPC 07-01) Ann Oncol. 2011;22(11):2466-2470.

10. Bailon O, Chouahnia K, Augier A, et al. Upfront association of carboplatin plus pemetrexed in patients with brain metastases of lung adenocarcinoma. Neuro Oncol. 2012;14(4):491-495.

11. Park SJ, Kim HT, Lee DH, et al. Efficacy of epidermal growth factor receptor tyrosine kinase inhibitors for brain metastasis in non-small cell lung cancer patients harboring either exon 19 or 21 mutation. Lung Cancer. 2012;77(3):556-560.

12. Su S, Wu YL. Clinical trials of tyrosine kinase inhibitors for lung cancer in China: a review. J Hematol Oncol. 2017;10(1):147.

13. Soon YY, Leong CN, Koh WY, Tham IW. EGFR tyrosine kinase inhibitors versus cranial radiation therapy for EGFR mutant non-small cell lung cancer with brain metastases: a systematic review and meta-analysis. Radiother Oncol. 2015;114(2):167-172.

14. Jiang T, Min W, Li Y, Yue Z, Wu C, Zhou C. Radiotherapy plus EGFRTKIs in non-small cell lung cancer patients with brain metastases: an update meta-analysis. Cancer Med. 2016;5(6):1055-1065.

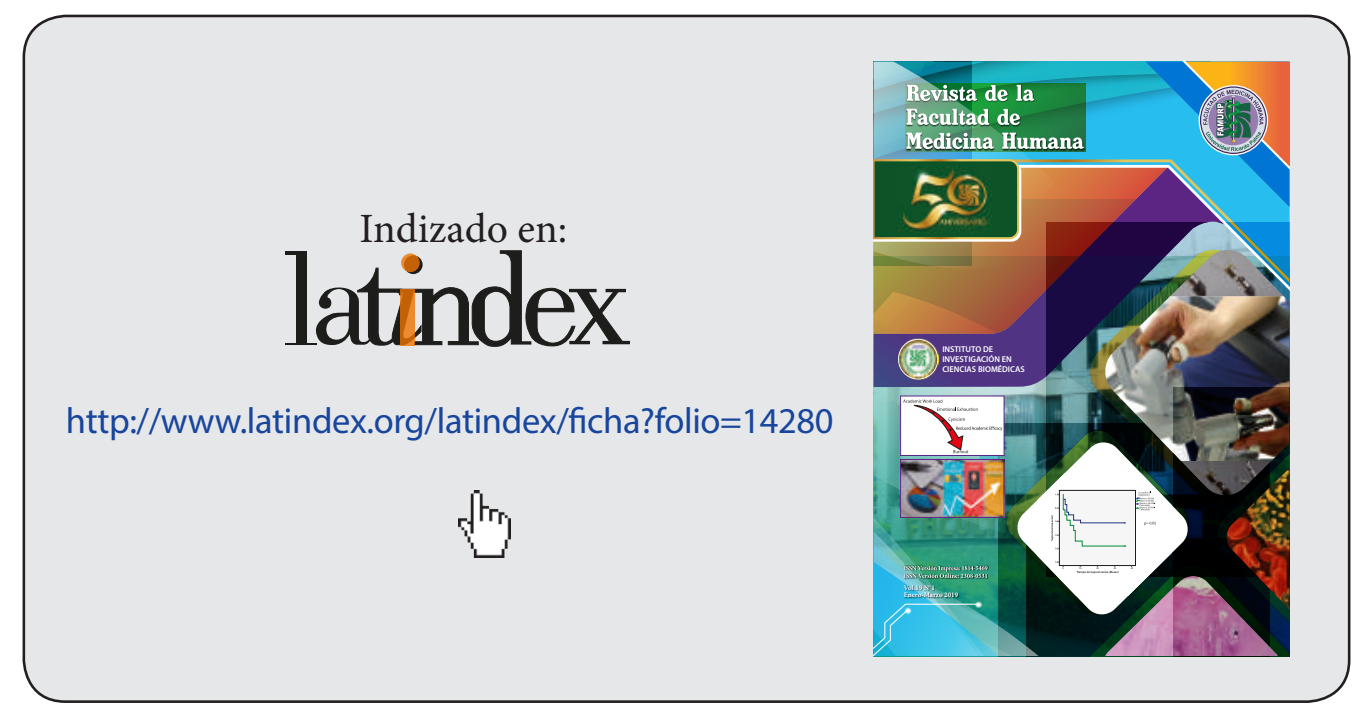

